International Mathematical Forum, 2, 2007, no. 46, 2295 - 2304

\title{
On Anti Fuzzy Left $h$-ideals in Hemirings
}

\author{
M. $\operatorname{Akram}^{1}$ \\ Punjab University College of Information Technology \\ University of the Punjab \\ Old Campus, Lahore-54000, Pakistan \\ m.akram@pucit.edu.pk \\ K. H. $\operatorname{Dar}^{2}$ \\ Govt. College University Lahore \\ Department of Mathematics \\ Katchery Road, Lahore-54000, Pakistan \\ prof_khdar@yahoo.com
}

\begin{abstract}
In this paper we apply the Biswas's idea of anti fuzzy subgroups to left $h$-ideals of hemirings. We introduce the notion of anti fuzzy left $h$-ideals in hemirings and investigate some of related properties. Relationship between fuzzy left $h$-ideals and anti fuzzy left $h$-ideals of hemirings is also given.
\end{abstract}

Mathematics Subject Classification: 16Y60, 13E05, 03G25

Keywords: Hemirings; (Anti fuzzy)left $h$-ideals; Normal anti fuzzy $h$ ideals

\section{Introduction}

Ideals of semirings play a central role in the structure theory and are useful for many purposes. However, they do not in general coincide with the usual ring ideals if $R$ is a ring and, for this reason, their use is somewhat limited in trying to obtain analogues of ring theorems for semirings. Indeed, many results in rings apparently have no analogues in semirings using only ideals. Henriksen defined in [2] a more restricted class of ideals in semirings, which is called the class of $k$-ideals, with the property that if the semiring $R$ is a ring

\footnotetext{
${ }^{1}$ The research work of first author is supported by PUCIT.

${ }^{2}$ The research work of second author is supported by HEC-Islamabad, Pakistan.
} 
then a complex in $R$ is a $k$-ideal if and only if it is a ring ideal. Another more restricted class of ideals has been given in hemirings by Iizuka [1]. However, in an additively commutative semiring $R$, ideals of a semiring coincide with "ideals" of a ring, provided that the semiring is a hemiring. We now call this ideal an $h$-ideal of the hemiring $R$. The properties of $h$-ideals and also $k$-ideals of hemirings were thoroughly investigated by La Torre in [3] and by using the $h$-ideals and $k$-ideals, La Torre established some analogous ring theorems for hemirings. There have been many applications of semirings and hemirings in different areas, such as social networks analysis [7], theory of automata and formal languages [5], idempotent analysis, discrete dynamical systems, fuzzy set theory, optimization [10].

The notion of fuzzy sets was first introduced by Zadeh [15]. Fuzzy set theory has been developed in many directions by many scholars and has evoked great interest among mathematicians working in different fields of mathematics, such as topological spaces, functional analysis, loop, group, ring, near ring, vector spaces, automation. There have been wide-ranging applications of the theory of fuzzy sets, from the design of robots and computer simulation to engineering and water resources planning. Rosenfeld [16] introduced the fuzzy sets in the realm of group theory. Since then many mathematicians have been involved in extending the concepts and results of abstract algebra to the broader frame work of the fuzzy setting. The notion of fuzzy $h$-ideals in hemirings was introduced in [13]. In this paper we apply the Biswas's idea of anti fuzzy subgroups to left $h$-ideals of hemirings. We introduce the notion of anti fuzzy left $h$-ideals in hemirings, and investigate some of their properties. Relationship between fuzzy left $h$-ideals and anti fuzzy left $h$-ideals of hemirings is also given.

\section{Preliminaries}

In this section we review some elementary aspects that are necessary for this paper:

An algebra $(R ;+, \cdot)$ is said to be a semiring if it satisfies the following conditions:

- $(R ;+)$ is a semigroup,

- $(R ; \cdot)$ is a semigroup,

- $(\forall a, b, c \in R)(a \cdot(b+c)=a \cdot b+a \cdot c$ and $(b+c) \cdot a=b \cdot a+c \cdot a)$.

A semiring $(R ;+, \cdot)$ is called a hemiring if

(H1) ' + ' is commutative and 
(H2) there exists an element $0 \in R$ such that 0 is the identity of $(R ;+)$ and the zero element of $(R ; \cdot)$. i.e., $0 \cdot a=a \cdot 0=0, \forall a \in R$.

A subset $I$ of a semiring $R$ is called a left ideal of $R$ if $I$ is closed under addition and $R I \subseteq I$. A left ideal of $R$ is called a left $k$-ideal of $R$ if $y, z \in I$ and $x \in R, x+y=z$ implies $x \in I$. A left $h$-ideal of a hemiring $R$ is defined to be a left ideal $A$ of $R$ such that

$$
(\forall x, z \in R)(\forall a, b \in A)(x+a+z=b+z \longrightarrow x \in A)
$$

Right $h$-ideals are defined similarly. A mapping $f: R_{1} \rightarrow R_{2}$ is said to be hemiring homomorphism of $R_{1}$ into $R_{2}$ if $f(x+y)=f(x)+f(y)$ and $f(x y)=f(x) f(y)$ for all $x, y \in R_{1}$. A mapping $\mu: X \rightarrow[0,1]$, where $X$ is an arbitrary nonempty set, is called a fuzzy set in $X$. For any fuzzy set $\mu$ in $X$ and any $\alpha \in[0,1]$ we define the set

$$
L(\mu ; \alpha)=\{x \in X \mid \mu(x) \leq \alpha\}
$$

which is called lower level cut of $\mu$. A fuzzy subset $\mu$ of a semiring $R$ is said to be a fuzzy left ideal of $R$ if

- $(\forall x, y \in R)(\mu(x+y) \geq \min \{\mu(x), \mu(y)\})$,

- $(\forall x, y \in R)(\mu(x y) \geq \mu(y))$.

Note that if $\mu$ is a fuzzy left ideal of a hemiring $R$, then $\mu(0) \geq \mu(x)$ for all $x \in R$. A fuzzy left ideal $\mu$ of a semiring $R$ is called a fuzzy left $k$-ideal of $R$ if for all $x, y, z \in R, x+y+z$ implies $\mu(x) \geq \min (\mu(y), \mu(z))$. A fuzzy left $h$-ideal of a hemiring $R$ is defined to be a fuzzy left ideal $\mu$ of $R$ such that

$$
(\forall x, z, a, b \in R)(x+a+z=b+z \longrightarrow \mu(x) \geq \min (\mu(a), \mu(b))) .
$$

Fuzzy right $h$-ideals are defined similarly.

\section{Anti fuzzy left $h$-ideals in hemirings}

Definition 3.1. A fuzzy subset $\mu$ of a hemiring $R$ is said to be an anti fuzzy left $h$-ideal of $R$ if

(a) $(\forall x, y \in R)(\mu(x+y) \leq \max (\mu(x), \mu(y)))$,

(b) $(\forall x, y \in R)(\mu(x y) \leq \mu(y))$,

(c) $(\forall x, z, a, b \in R)(x+a+z=b+z \longrightarrow \mu(x) \leq \max (\mu(a), \mu(b)))$. 
Example 3.2. Let $R=\{0,1,2,3,4\}$ be a hemiring with zero multiplication and addition defined by the following table.

\begin{tabular}{l|lllll} 
& 0 & 1 & 2 & 3 & 4 \\
\hline 0 & 0 & 1 & 2 & 3 & 4 \\
1 & 1 & 1 & 4 & 4 & 4 \\
2 & 2 & 4 & 4 & 4 & 4 \\
3 & 3 & 4 & 4 & 4 & 4 \\
4 & 4 & 4 & 4 & 4 & 4
\end{tabular}

We define a fuzzy set $\mu: R \rightarrow[0,1]$ by letting $\mu(0)=t_{1}$ and $\mu(x)=t_{2}$ for all $x \neq 0, t_{1}<t_{2}$. By routine computations, we can also easily check that $\mu$ is an anti fuzzy $h$-ideal of hemiring $R$.

Proposition 3.3. Let $R$ be a hemiring and $\mu$ be a fuzzy set in $R$. Then $\mu$ is an anti fuzzy $h$-ideal in $R$ if and only $\mu^{c}$ is a fuzzy left $h$-ideal in $R$.

Proof. Let $\mu$ be an anti fuzzy left $h$-ideal in $R$. For $x, y \in R$, we have

$$
\begin{gathered}
\mu^{c}(x+y)=1-\mu(x+y) \geq 1-\max (\mu(x), \mu(y)) \\
=\min (1-\mu(x), 1-\mu(y))=\min \left(\mu^{c}(x), \mu^{c}(y)\right), \\
\mu^{c}(x y)=1-\mu(x y) \geq 1-\mu(y)=\mu^{c}(y) .
\end{gathered}
$$

Let $x, z, a, b \in R$ be such that $x+a+z=b+z$. Then

$$
\begin{aligned}
\mu^{c}(x) & =1-\mu(x) \geq 1-\max (\mu(a), \mu(b)) \\
& =\min (1-\mu(a), 1-\mu(b))=\min \left(\mu^{c}(a), \mu^{c}(b)\right) .
\end{aligned}
$$

Hence $\mu^{c}$ is a fuzzy left $h$-ideal of $R$.

Conversely, let $\mu^{c}$ be a fuzzy left $h$-ideal in $R$. For $x, y \in R$, we have

$$
\begin{aligned}
\mu(x+y) & =1-\mu^{c}(x+y) \leq 1-\min \left(\mu^{c}(x), \mu^{c}(y)\right) \\
& =\max (\mu(x), \mu(y)), \\
\mu(x y) & =1-\mu^{c}(x y) \leq 1-\mu^{c}(y)=\mu(y) .
\end{aligned}
$$

Let $x, z, a, b \in R$ be such that $x+a+z=b+z$. Then

$$
\begin{aligned}
\mu(x) & =1-\mu^{c}(x) \leq 1-\min \left(\mu^{c}(a), \mu^{c}(b)\right) \\
& =\max (\mu(a), \mu(b)) .
\end{aligned}
$$

Hence $\mu$ is an anti fuzzy left $h$-ideal of $R$. 
Theorem 3.4. Let $\mu$ be an anti fuzzy left $h$-ideal in a hemiring $R$ such that $L(\mu ; \alpha)$ is a left $h$-ideal of $R$ for each $\alpha \in \operatorname{Im}(\mu), \alpha \in[0,1]$. Then $\mu$ is an anti fuzzy left $h$-ideal of $R$.

Proof. Let $x, y \in R$ be such that $\mu(x)=\alpha_{1}$ and $\mu(y)=\alpha_{2}$. Then $x+y \in$ $L(\mu ; \alpha)$. Without loss of generality, we may assume that $\alpha_{1} \geq \alpha_{2}$. It follows that $L\left(\mu ; \alpha_{2}\right) \subseteq L\left(\mu ; \alpha_{1}\right)$ so that $x \in L\left(\mu ; \alpha_{1}\right)$ and $y \in L\left(\mu ; \alpha_{2}\right)$. Since $L\left(\mu ; \alpha_{1}\right)$ is a left $h$-ideal of $R$, we have $x+y \in L\left(\mu ; \alpha_{1}\right)$. Thus

$$
\begin{gathered}
\mu(x+y) \leq \alpha_{1}=\max (\mu(x), \mu(y)), \\
\mu(x y) \leq \alpha_{1}=\mu(y) .
\end{gathered}
$$

Let $x, z, a, b \in R$ be such that $x+a+z=b+z$. Then $\mu(x) \leq \alpha_{1}=$ $\max \{\mu(a), \mu(b)\}$. This shows that $\mu$ is an anti fuzzy left $h$-ideal of $R$.

Corollary 3.5. Let $\mu$ be an anti-fuzzy left $h$-ideal of $R$. Then $\mu$ is an antifuzzy left $h$-ideal of $R$ if and only if $L(\mu ; \alpha)$ is left $h$-ideal of $R$ for every $\alpha \in[\mu(0), 1]$ with $\alpha \in[0,1]$.

Theorem 3.6. Let $\mu$ be an anti fuzzy set in a hemiring $R$. Then two lower level subsets $L\left(\mu ; t_{1}\right)$ and $L\left(\mu ; t_{2}\right)\left(t_{1}<t_{2}\right)$ are equal if and only if there is no $x \in R$ such that $t_{1}<\mu(x) \leq t_{2}$.

Proof. From definition of $L(\mu ; \alpha)$, it follows that $L(\mu ; t)=\mu^{-1}([\mu(0), t])$ for $t \in[0,1]$.

Let $t_{1}, t_{2} \in[0,1]$ be such that $t_{1}<t_{2}$. Then

$$
\begin{aligned}
L\left(\mu ; t_{1}\right) & =L\left(\mu ; t_{2}\right) \\
& \Leftrightarrow \mu^{-1}\left(\left[\mu(0) ; t_{1}\right]\right)=\mu^{-1}\left(\left[\mu(0) ; t_{2}\right]\right) \\
& \Leftrightarrow \mu^{-1}\left(t_{1}, t_{2}\right)=\emptyset \\
& \Leftrightarrow \text { there } i s \text { no } x \in R \text { such that } t_{1}<\mu(x) \leq t_{2} .
\end{aligned}
$$

This complete the proof.

Definition 3.7. A left $h$-ideal $A$ of hemiring $R$ is said to be characteristic if $f(A)=A$, for all $f \in \operatorname{Aut}(R)$, where $\operatorname{Aut}(R)$ is the set of all automorphisms of $R$. Anti fuzzy left $h$-ideal $\mu$ of hemiring $R$ is said to be anti fuzzy characteristic if $\mu^{f}(x)=\mu(x)$, for all $x \in R$ and $f \in \operatorname{Aut}(R)$.

Lemma 3.8. Let $\mu$ be an anti fuzzy left h-ideal of a hemiring $R$ and let $x \in R$. Then $\mu(x)=s$ if and only if $x \in L(\mu ; s)$ and $x \notin L(\mu ; t)$, for all $s>t$.

Proof. Straightforward. 
Theorem 3.9. Let $\mu$ be an anti fuzzy h-ideal of a hemiring $R$. Then each level left $h$-ideal of $\mu$ is characteristic if and only if $\mu$ is an anti fuzzy characteristic.

Proof. Suppose that $\mu$ is anti fuzzy characteristic and let $s \in \operatorname{Im}(\mu), f \in \operatorname{Aut}(R)$ and $x \in L(\mu ; s)$. Then

$$
\mu^{f}(x)=\mu(x) \leq s \Rightarrow \mu(f(x)) \leq s \Rightarrow f(x) \in L(\mu ; s) .
$$

Thus

$$
f(L(\mu ; s)) \subseteq L(\mu ; s) .
$$

Let $x \in L(\mu ; s)$ and $y \in R$ such that $f(y)=x$. Then

$$
\begin{aligned}
\mu(y) & =\mu^{f}(y)=\mu(f(y))=\mu(x) \leq s \\
& \Rightarrow y \in L(\mu ; s) \text { so that } x=f(y) \in L(\mu ; s) .
\end{aligned}
$$

Consequently, $L(\mu ; s) \subseteq f(L(\mu ; s))$. Hence $f(L(\mu ; s))=L(\mu ; s)$, i.e., $L(\mu ; s)$ is characteristic. Conversely, suppose that each level $h$-ideal of $\mu$ is characteristic and let $x \in R, f \in A u t(R)$ and $\mu(x)=s$. Then, by virtue of Lemma 3.8, $x \in L(\mu ; s)$ and $x \notin L(\mu ; t)$, for all $s>t$. It follows from the assumption that $f(x) \in f(L(\mu ; s))=L(\mu ; s)$, so that $\left.\mu^{f}(x)=\mu(f(x))\right) \leq s$. Let $t=\mu^{f}(x)$ and assume that $s>t$. Then $f(x) \in L(\mu ; t)=f(L(\mu ; t))$, which implies from the injectivity of $f$ that $x \in L(\mu ; t)$, a contradiction. Hence $\mu^{f}(x)=\mu(f(x))=$ $s=\mu(x)$ showing that $\mu$ is an anti fuzzy characteristic.

Theorem 3.10. Let $f: R_{1} \rightarrow R_{2}$ be an epimorphism of hemirings. If $\nu$ is an anti-fuzzy left $h$-ideal of $R_{2}$ and $\mu$ is the pre-image of $\nu$ under $f$. Then $\mu$ is an anti fuzzy left $h$-ideal of $R_{1}$.

Proof. For any $x, y \in R_{1}$, we have

$$
\begin{aligned}
\mu(x+y) & =\nu(f(x+y))=\nu(f(x)+f(y)) \\
& \leq \max (\nu(f(x)), \nu(f(y)))=\max (\mu(x), \mu(y)), \\
\mu(x y)= & \nu(f(x y))=\nu(f(x) f(y)) \leq \nu(f(y))=\mu(y) .
\end{aligned}
$$

Let $x, z, a, b \in R_{1}$ be such that $x+a+z=b+z$. Then, we have

$$
\mu(x)=\nu(f(x)) \leq \max (\nu(f(a)), \nu(f(b)))=\max (\mu(a), \mu(b)) .
$$

Hence $\mu$ is an anti fuzzy left $h$-ideal of $R_{1}$.

Definition 3.11. Let $R_{1}$ and $R_{2}$ be two hemirings and $f$ be a function of $R_{1}$ into $R_{2}$. If $\mu$ is a fuzzy set in $R_{2}$, then the preimage of $\mu$ under $f$ is the fuzzy set in $R_{1}$ defined by

$$
f^{-1}(\mu)(x)=\mu(f(x)) \quad \forall x \in R_{1} .
$$


Theorem 3.12. Let $f: R_{1} \rightarrow R_{2}$ be an onto homomorphism of hemirings. If $\mu$ is an anti fuzzy left $h$-ideal of $R_{2}$, then $f^{-1}(\mu)$ is an anti fuzzy left $h$-ideal of $R_{1}$.

Proof. Let $x_{1}, x_{2} \in R_{1}$, then we have

$$
\begin{gathered}
f^{-1}(\mu)\left(x_{1}+x_{2}\right)=\mu\left(f\left(x_{1}\right)+f\left(x_{2}\right)\right) \leq \max \left(\mu\left(f\left(x_{1}\right)\right), \mu\left(f\left(x_{2}\right)\right)\right) \\
=\max \left(f^{-1}(\mu)\left(x_{1}\right), f^{-1}(\mu)\left(x_{2}\right)\right) \\
\\
f^{-1}(\mu)\left(x_{1} x_{2}\right)=\mu\left(f\left(x_{1}\right) f\left(x_{2}\right)\right) \leq \mu\left(f\left(x_{2}\right)\right) \\
=f^{-1}(\mu)\left(x_{2}\right) .
\end{gathered}
$$

Let $x, z, a, b \in R_{1}$ be such that $x+a+z=b+z$. Then, we have

$$
\begin{aligned}
f^{-1}(\mu)(x) & =\mu(f(x)) \leq \max (\mu(f(a)), \mu(f(b))) \\
& =\max \left(f^{-1}(\mu)(a), f^{-1}(\mu)(b)\right) .
\end{aligned}
$$

Hence $f^{-1}(\mu)$ is an anti fuzzy left $h$-ideal of $R_{1}$.

Definition 3.13. Let $R_{1}$ and $R_{2}$ be any sets and let $f: R_{1} \rightarrow R_{2}$ be any function. A fuzzy subset $\mu$ of $R_{1}$ is called f-invariant if $f(x)=f(y)$ implies $\mu(x)=\mu(y), x, y \in R_{1}$.

Theorem 3.14. Let $f: R_{1} \rightarrow R_{2}$ be an epimorphism of hemirings. Let $\mu$ be an $f$-invariant anti fuzzy left $h$-ideal of $R_{1}$. Then $f(\mu)$ is an anti fuzzy left $h$-ideal of $R_{2}$.

Proof. Let $x, y \in R_{2}$. Then there exist $a, b \in R_{1}$ such that $f(a)=x$ and $f(b)=y$, then $x+y=f(a+b)$ and $x y=f(a b)$. Since $\mu$ is $f$-invariant,

$$
\begin{aligned}
f(\mu)(x+y) & =\mu(x+y) \leq \max (\mu(a), \mu(b)) \\
& =\max (f(\mu)(x), f(\mu)(y)), \\
f(\mu)(x y) & =\mu(a b) \leq \mu(b)=f(\mu)(y) .
\end{aligned}
$$

Let $x, z, a, b \in R_{2}$ be such that $x+a+z=b+z$, then there exist $\dot{x}, \dot{z} ., \dot{a}, \dot{b}$ such that $f(\dot{x})=x, f(\dot{y})=y, f(\dot{a})=a$ and $f(\hat{b})=b$. Since $\mu$ is $f$-invariant,

$$
\begin{aligned}
f(\mu)(x) & =\mu(x) \leq \max (\mu(\dot{a}), \mu(\dot{b})) \\
& =\max (f(\mu)(a), f(\mu)(b)) .
\end{aligned}
$$

Hence $f(\mu)$ is an anti fuzzy left $h$-ideal of $R_{2}$. 
Definition 3.15. A fuzzy left $h$-ideal $\mu$ of a hemiring $R$ is said to be normal if there exist $x \in R$ such that $\mu(x)=1$. Note that if $\mu$ is a normal anti fuzzy left $h$-ideal of $R$, then $\mu(0)=1$, and hence $\mu$ is normal if and only if $\mu(0)=1$.

Theorem 3.16. Let $\mu$ be an anti fuzzy left $h$ ideal of a hemiring $R$. Let $\mu^{+}$ be a fuzzy set in $R$ defined by $\mu^{+}(x)=\mu(x)+1-\mu(0)$ for all $x \in R$. Then $\mu^{+}$ is a normal anti fuzzy left $h$-ideal of $R$ which contains $\mu$.

Proof. For any $x, y \in R$, we have $\mu^{+}(x)=\mu(0)+1-\mu(0)=1$ and

$$
\begin{aligned}
& \mu^{+}(x+y)=\mu(x+y)+1-\mu(0) \leq \max (\mu(x), \mu(y))+1-\mu(0) \\
&=\max (\mu(x)+1-\mu(0), \mu(y)+1-\mu(0))=\max \left(\mu^{+}(x), \mu^{+}(y)\right), \\
& \mu^{+}(x y)=\mu(x y)+1-\mu(0) \leq \mu(y)+1-\mu(0)=\mu^{+}(y) .
\end{aligned}
$$

This shows that $\mu^{+}$is an anti fuzzy left ideal of $R$. Let $a, b, x, z \in R$ be such that $x+a+z=b+z$. Then

$$
\begin{aligned}
\mu^{+}(x) & =\mu(x)+1-\mu(0) \leq \max (\mu(a), \mu(b))+1-\mu(0) \\
& =\max (\mu(a)+1-\mu(0), \mu(b)+1-\mu(0))=\max \left(\mu^{+}(a), \mu^{+}(b)\right) .
\end{aligned}
$$

Hence $\mu^{+}$is a normal anti fuzzy left $h$-ideal of hemiring of $R$. Clearly, $\mu \subset \mu^{+}$. This ends the proof.

Definition 3.17. Let $\mathbb{N}(R)$ denote the set of all normal anti fuzzy left $h$-ideals of $R$. Note that $\mathbb{N}(R)$ is a poset under the set inclusion. A fuzzy set $\mu$ in a hemiring $R$ is called a maximal anti fuzzy left $h$-ideal of $R$ if it is non-constant and $\mu^{+}$is a maximal element of $(\mathbb{N}(R), \subseteq)$.

Theorem 3.18. Let $\mu \in \mathbb{N}(R)$ be non-constant such that it is a maximal element of $(\mathbb{N}(R), \subseteq)$. Then it takes only two values $\{0,1\}$.

Proof. Since $\mu$ is normal, $\mu(0)=1$. We claim that $\mu(x)=0$. If not, then there exists $x_{0} \in R$ such that $0<\mu\left(x_{0}\right)<1$. Define on $R$ a fuzzy set $\nu$ by putting $\nu(x)=\frac{1}{2}\left(\mu(x)+\mu\left(x_{0}\right)\right)$ for each $x \in R$. Then clearly $\nu$ is well-defined and for all $x, y \in R$, we have

$$
\begin{aligned}
\nu(x+y) & =\frac{1}{2} \mu(x+y)+\frac{1}{2} \mu\left(x_{0}\right) \leq \frac{1}{2}\left(\max \{\mu(x), \mu(y)\}+\mu\left(x_{0}\right)\right) \\
& =\max \left\{\frac{1}{2}\left(\mu(x)+\mu\left(x_{0}\right)\right), \frac{1}{2}\left(\mu(y)+\mu\left(x_{0}\right)\right)\right\}=\max \{\nu(x), \nu(y)\}
\end{aligned}
$$




$$
\begin{aligned}
\nu(x y) & =\frac{1}{2} \mu(x y)+\frac{1}{2} \mu\left(x_{0}\right) \leq \frac{1}{2}\left(\mu(y)+\mu\left(x_{0}\right)\right) \\
& =\nu(y) .
\end{aligned}
$$

Thus $\nu$ is an anti fuzzy left ideal of $R$. Let $a, b, x, z \in R$ be such that $x+a+z=b+z$. Then

$$
\begin{aligned}
\nu(x) & =\frac{1}{2} \mu(x)+\frac{1}{2} \mu\left(x_{0}\right) \leq \frac{1}{2}\left(\max \left\{\mu(a), \mu(b)+\mu\left(x_{0}\right)\right\}\right) \\
& =\max \left\{\frac{1}{2}\left(\mu(a)+\mu\left(x_{0}\right)\right), \frac{1}{2}\left(\mu(b)+\mu\left(x_{0}\right)\right)\right\}=\max (\nu(a), \nu(b)) .
\end{aligned}
$$

Hence $\nu$ is an anti fuzzy left $h$-ideal of $R$. By Theorem 3.16, $\nu^{+}$is a maximal anti fuzzy left $h$-ideal of $R$. Note that

$$
\begin{gathered}
\nu^{+}(x)=\nu(x)+1-\nu(0)=\frac{1}{2}\left(\mu(x)+\mu\left(x_{0}\right)\right)+1-\frac{1}{2}\left(1+\mu\left(x_{0}\right)\right) \\
=\frac{1}{2}\left(\mu\left(x_{0}\right)+1\right)=\nu\left(x_{0}\right),
\end{gathered}
$$

and $\nu^{+}\left(x_{0}\right)<1=\nu^{+}(0)$. Hence $\nu^{+}$is a non-constant, and $\mu$ is not a maximal element of $\mathbb{N}(R)$. This is a contradiction. This ends the proof.

\section{References}

[1] K. Iizuka, On the Jacobson radical of a semiring, Tohoku Math. J., 11 (2) (1959) 409-421.

[2] M. Henriksen, Ideals in semirings with commutative addition, Am. Math. Soc. Notices, 6 (1958) 3-21.

[3] D. R. La Torre, On h-ideals and k-ideals in hemirings, Publ. Math. Debrecen, 12 (1965), 219- 226.

[4] D. M. Olson, A note on the homomorphism theorem for hemirings, IJMMS, 1 (1978), 439-445.

[5] A. W. Aho and J.D. Ullman, Introduction to automata theory, languages and computation, Addison-Wesley, Reading, MA, 1979.

[6] K. Hashiguchi, Limitedness theorem for finite automata with distance functions, J. Comput. Syst. Sci. 24 (1982) 233-244. 
[7] V. Batagelj, Semirings for social network analysis, J. Math. Sociology, 19(1)(1994)53-68.

[8] S. Jonathan and J. S. Golan, Semirings and their applications, Kluwer Academic Publishers, 1999.

[9] T. K. Dutta and B. K. Biswas, Fuzzy k-ideals of semirings, Bull. Calcutta Math. Soc. 87 (1995), 91-96. 7.

[10] S. Ghosh, Fuzzy k-ideals of semirings, Fuzzy sets and Systems, 95(1998),103-108.

[11] C. B. Kim and M. Park, k-fuzzy ideals in semirings, Fuzzy sets and Systems, 81(1996),281-286.

[12] W. J. Liu, Fuzzy invariants subgroups and fuzzy ideals, Fuzzy Sets and Systems, 8 (1987), 133-139.

[13] Y. B. Jun, M. A. Ozturk and S. Z. Song, On fuzzy h-ideals in hemirings, Info. Scien., 162(2004), 211-226.

[14] R. Biswas, Fuzzy subgroups and anti fuzzy subgroups, Fuzzy Sets Syst. 44 (1990) 121-124.

[15] L. A. Zadeh, Fuzzy sets, Information Control, 8(1965) 338-353.

[16] A. Rosenfeld, Fuzzy groups, J. Math. Anal. Appl. 35 (1971), 512-517.

Received: January 31, 2006 